\title{
Crustaceen-Plankton des Vierwaldstättersees Grössenfraktionierung und Abundanzdynamik in den Jahren 1976 bis 1979
}

\author{
von Bürgi, H. R. und Egli, B. \\ Manuskript eingegangen am 30. Oktober 1984
}

\begin{abstract}
In order to create a common comparative biomass basis for the biological interactions between zooplankton and phytoplankton, zooplankton was quantitavely sampled at close intervals, counted and measured at determined development stages. The volumetric values calculated according to stereometric formulas do not indicate an important variability over the years. Significant volume increases of adult crustaceans were observed only from March till May. Comparisons with past plankton analyses revealed a rapid increase of the herbivorous crustaceans, i.e. by a factor of five; while simultaneously, the favourite food (nannoplankton) only increased from $3.1 \mathrm{~g} / \mathrm{m}^{2}$ to $5.5 \mathrm{~g} / \mathrm{m}^{2}$ wet weight.
\end{abstract}

\section{Zielsetzung}

Das Studium integraler limnischer Ökosysteme erfordert detaillierte Kenntnisse der Entwicklung von Phyto- und Zooplanktongruppen. Die entsprechenden Daten müssen in einer einheitlichen und mit andern Parametern vergleichbaren Form (z. B. auf der Basis von Biomasse) vorliegen.

Mit der heutigen Analysentechnik lassen sich aus einem Zooplanktonfang einzelne Arten und sogar deren Stadien aussortieren und mit Hilfe von Biomasse-Ersatzparametern (partikulärer organischer Kohlenstoff, ATP, Energie-Inhalt, Trockengewicht) quantifizieren. Beim Phytoplankton liefern diese Methoden dagegen nur pauschale Summenwerte, ohne weitere Differenzierung. Wo eine weitere Aufteilung nötig ist, kommen die bekannten indirekten Methoden der Biomassebestimmung anhand von stereometrischen Volumenberechnungen in Kombination mit Zählungen nach Utermöhl [12] zur Anwendung. Die Differenzierung der Biomasse nach Arten bringt allerdings auch grössere systematische Fehler mit sich: Nach Interkalibrierungstests liegen 95\% der Resultate innerhalb einer 30\%-Abweichung vom Mittelwert [7]. Berechnungsgrundlagen und Voluminawerte existieren auch für Zooplanktonarten $[5,8,9,10]$. Die Güte der Berechnung hängt im wesentlichen davon ab, wie genau die Variabilität der Körperdimensionen der Organismen während eines längeren Zeitraumes berücksichtigt werden kann. Das Ziel der vorliegenden Arbeit besteht darin, die Variabilität für die 
häufigeren Crustaceenarten in verschiedenen Phasen der Entwicklung zu messen. Beim Vergleichen mit früheren Zooplankton-Untersuchungen liefern die neueren Zählungen wertvolle Hinweise auf die Auswirkungen der inzwischen erfolgten trophischen Veränderungen des Vierwaldstättersees.

\section{Material und Methoden}

Die Probenahmen erfolgten mit Zwillingsnetzen [3] mit $95 \mu \mathrm{m}$ Maschenweite, je $1 \mathrm{dm}^{2}$ Eintrittsöffnung mit $50 \mathrm{dm}^{2}$ Netzfläche. Ein einzelner Fang bestand aus fünf horizontal versetzten Vertikalzügen von $110 \mathrm{~m}$ Tiefe bis zur Oberfläche. Probestelle: Grösste Tiefe (111 m) im Kreuztrichterbecken des Vierwaldstättersees (Nähe Luzern). Der Probenahmerhythmus wurde der Entwicklung des Zooplanktons angepasst; normalerweise betrug das Zeitintervall 8 bis 14 Tage.

Die Crustaceen wurden mit Formol konserviert (1:20), differenziert ausgezählt und in $4 \%$ Formollösung mit Glycerinzusatz aufbewahrt. Für die Analyse der Grössenverteilung wurden in gezielten Zeitabständen (etwa $4 \times$ pro Jahr) 10 bis 50 Individuen aus jeder Tiergruppe ausgemessen. Daphnien und Bosminen wurden als Ellipsoid, Copepoden als Rotationsellipsoid berechnet, wobei die gemessenen Achsen den grössten Abmessungen des Aussenskelettes ohne Abdomen, Fortsätze oder Helmspitze entsprachen. (siehe Umriss-Skizze). Die Volumina wurden in Nassgewicht umgerechnet, wobei
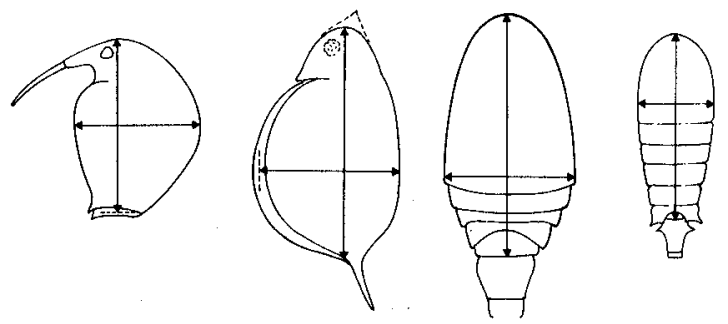

$10^{6} \mu \mathrm{m}^{3}=1 \mu \mathrm{g}$ gesetzt wurden.

Die Messungen konnten nicht unmittelbar nach der Probenahme oder nach einem festen Zeitplan erfolgen, weshalb die Proben bis zu ihrer Verarbeitung unterschiedlich lange aufbewahrt wurden. Es musste deshalb zunächst geprüft werden, ob sich durch Fixierung und Lagerung das Volumen verändert. $\mathrm{Zu}$ diesem Zweck wurden 60 Eudiaptomus gracilis ad. lebend (narkotisiert mit MS 222), unmittelbar nach der Formolfixierung und erneut nach $1 \mathrm{Tag}$, nach 4 Tagen und nach 1 Jahr ausgemessen. Diese Analysen ergaben keine signifikanten Veränderungen der berücksichtigten Körperdimensionen.

\section{Resultate}

\subsection{Bosmina spp. (Abb. 1, 2)}

Im untersuchten Zeitraum laufen die Vermehrungsphasen jahreszyklisch ab. Die Entwicklung beginnt Ende März und endet mehr oder weniger abrupt im Juni/Juli. Die Ausdehnung der Maxima hängt von der Dichte der Startpopulation im Februar/März ab; eine entsprechende Korrelation ist deutlich. So sind in den Frühjahrsphasen 1976 
Artenliste und Zählkategorien

Aus zeitlichen und technischen Gründen (z.B. Fehlen von stabilen Unterscheidungskriterien, insbesondere bei den Cladoceren) wurden z.T. umfassendere Zählkategorien gebildet:

\begin{tabular}{llll} 
Gattung/Art & Zählkategorie & dargestellt in \\
\cline { 2 - 3 } & Artspezifisch & zusammengefasst & \\
\hline
\end{tabular}

Diaphanosoma brachyurum juv.

Eubosmina coregoni

Bosmina longirostris

Daphnia hyalina

Daphnia galeata

Daphnia cucullata

Bythotrephes longimanus

Leptodora kindtii

Eudiaptomus gracilis

Mixodiaptomus laciniatus

Cyclops vicinus

Cyclops abyssorum

Cyclops bohater

Megacyclops gigas

Mesocyclops leuckarti juv.
ad.
Abb. 1

Abb. 2

Abb. 3

Abb. 4

*)

juv.

ad.

juv.

ad.

ad. 0

ad. 오, 우 ov

ad. 0, 우

ad. 우 ov

ad.

*)

Abb. 6

Abb. 6

Abb. 5

*)

Abb. 11

Nauplien

Abb. 7

Abb. 7

Abb. 8

Abb. 8

*)

Abb. 9

Abb. 10

*)

ad. 9,9 ov

ad. $\delta$

ad. 요 아 ov

*) seltenere Formen, wurden nur in Tab. 1 (Maxima ad. Tiere) berücksichtigt.

und 1977 als Restbestände der Winterpopulation deutlich höhere Individuendichten vorhanden, welche nach einer exponentiellen Vermehrungsphase in frühen Maxima gipfeln, während in den folgenden Jahren eine rund 5 mal kleinere Ausgangspopulation entsprechend geringere - und meist später auftretende - Maxima bewirkt.

Die Grössenverteilung der juvenilen Bosminen zeigt ein stabiles Verteilungsbild mit starker Dominanz der kleinen Individuen. Die Wahrscheinlichkeit, das Adultstadium $\mathrm{zu}$ erreichen beträgt $\mathrm{p}<0,1$. Die eiertragenden Bosminen nehmen im Laufe des Entwicklungsschubes im April/Mai leicht an Grösse zu, während die juvenilen Stadien im Mittel kleiner werden (Abb. 12). Diese Divergenz kann nicht mit einer Ablösung der 
beiden Bosmina-Arten erklärt werden (Eubosmina coregoni ist stets 6 bis 10 mal häufiger als B. longirostris) möglicherweise handelt es sich hier um Temporalvariationen, wie sie auch für andere Seen beschrieben wurden.

\subsection{Daphnia spp. (Abb. 3, 4)}

Die Jahresdynamik der Daphnien gleicht jener von Bosmina, wobei aber der Beginn der exponentiellen Vermehrungsphase (Ende April) rund einen Monat später erfolgt. Nach dem Zusammenbruch der Population infolge «Overgrazing» erscheinen aber regelmässige Wachstumsspitzen im Sommer und Herbst. Das Verhältnis von erwachsenen zu juvenilen Individuen erhöht sich im Laufe des Kalenderjahres. Die Grössenklassierung zeigt nur geringe Schwankungen; bei den juvenilen Formen sind in der Hauptvegetationsperiode rund 50 bis $70 \%$ aller Individuen kleiner als $100 \mathrm{Mio}^{3}$, während die erwachsenen Tiere grösstenteils ein Volumen zwischen 400 und 600 Mio $\mu^{3}$ besitzen. Die Wahrscheinlichkeit, dass die kleinsten Jungtiere ihre Biomasse verdoppeln können, liegt oft unter $p=0,2$. Erst im Winter erscheinen grössere Jugendformen in höheren Anteilen.

Innerhalb der 4 Berichtsjahre sind keine Änderungen zu beobachten. Die Volumina der juvenilen und adulten Tiere sind ähnlich wie bei Bosmina gegenläufig (Abb.12): Von Februar bis Juni nehmen die erwachsenen Tiere durchschnittlich von $460 \mathrm{Mio} \mu \mathrm{m}^{3} \mathrm{um}$

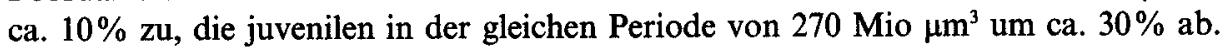

\subsection{Eudiaptomus gracilis (Abb. 5, 6)}

Bei Eudiaptomus sind die einzelnen Generationen anhand der vorliegenden Zahlen nur schwer auseinanderzuhalten. Mittelholzer fand 1968 noch 4 Generationen [6]. Alle Ent-

Abb.1-11. Abundanzdynamik und Grössenfraktionierung der häufigsten Crustaceen des Vierwaldstättersee-Planktons.

Die Grössenverteilung gibt den prozentualen Anteil der Volumenklassen auf $2.5 \%$ genau an. Die Grenzen der Grössenklassen sind in $\mu \mathrm{g} /$ Individuum (entspricht $10^{6} \mu \mathrm{m}^{3}$ der Dichte 1.0) angegeben. Bei der nach Männchen und Weibchen gegliederten Grössenfraktionierung adulter Copepoden gelten üblicherweise verschiedene Klassenbreiten. Im Juni 1978 konnte infolge eines technischen Defektes anstelle von fünf Netzzügen nur einer durchgeführt werden, die entsprechenden Werte $\left(\mathrm{Ind} / \mathrm{m}^{2}\right)$ sind in den Abbildungen gestrichelt dargestellt. Die

Pfeile markieren den Zeitpunkt der Probenahme für die Grössenfraktionierung.

Figures 1-11. Abundancedynamics and size fractioning of the most numerous Crustaceans in the Lake Lucerne plankton.

The size distribution gives the fraction of the size classes within $2.5 \%$ accuracy. The boundaries of the size classes are given in $\mu \mathrm{g} /$ Individual (this equals $10^{6} \mu \mathrm{m}^{3}$ at the spec. density of 1.0 ) Usually classes with differing width are given for male and female adult Copepods. Due to a technical defect, only one, instead of fife net samplings was taken in June 1978; the values (Individ. $/ \mathrm{m}^{2}$ ) in the figures are drawn with dotted lines. The arrows mark the sampling time of the size fraction samples. 

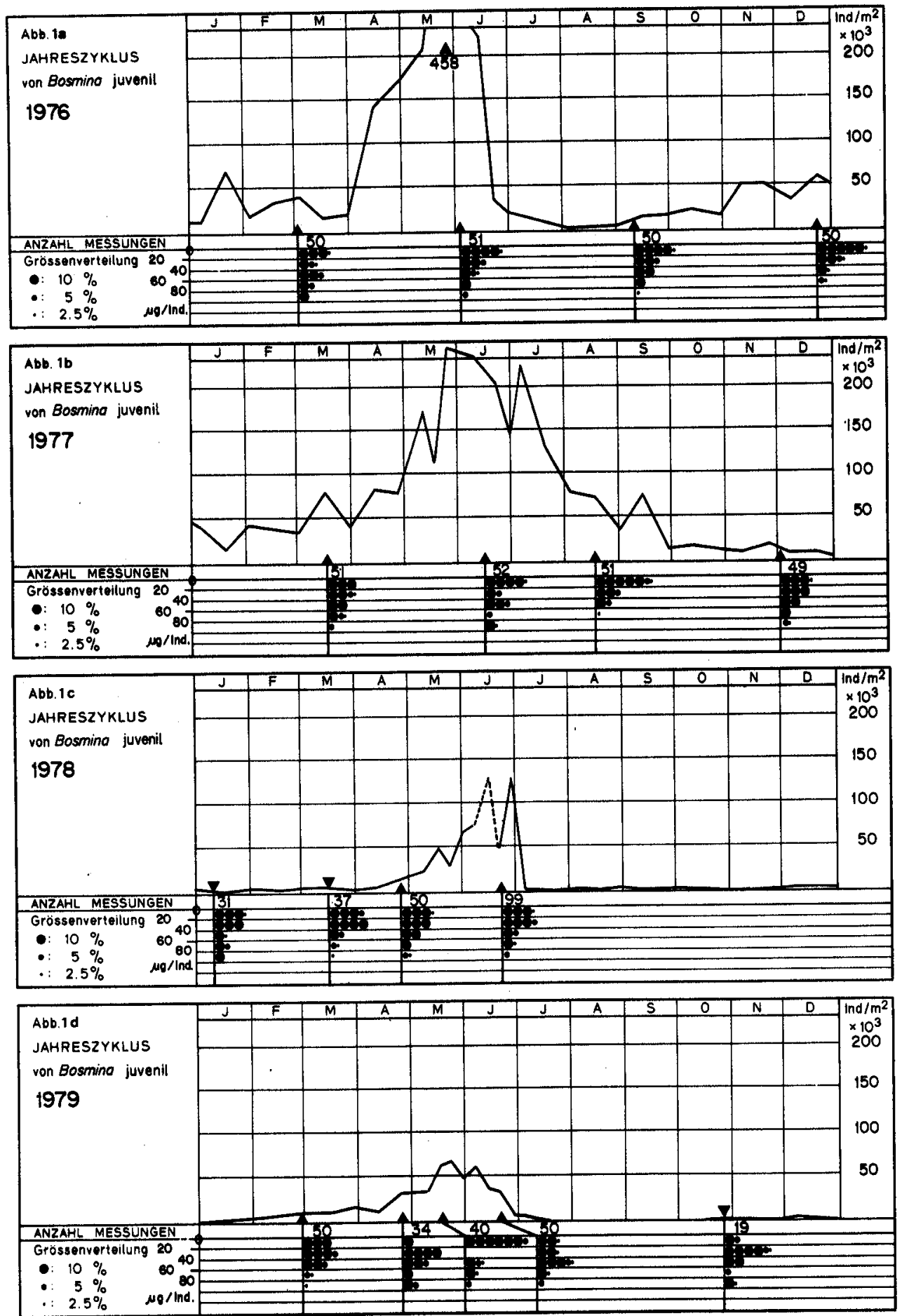

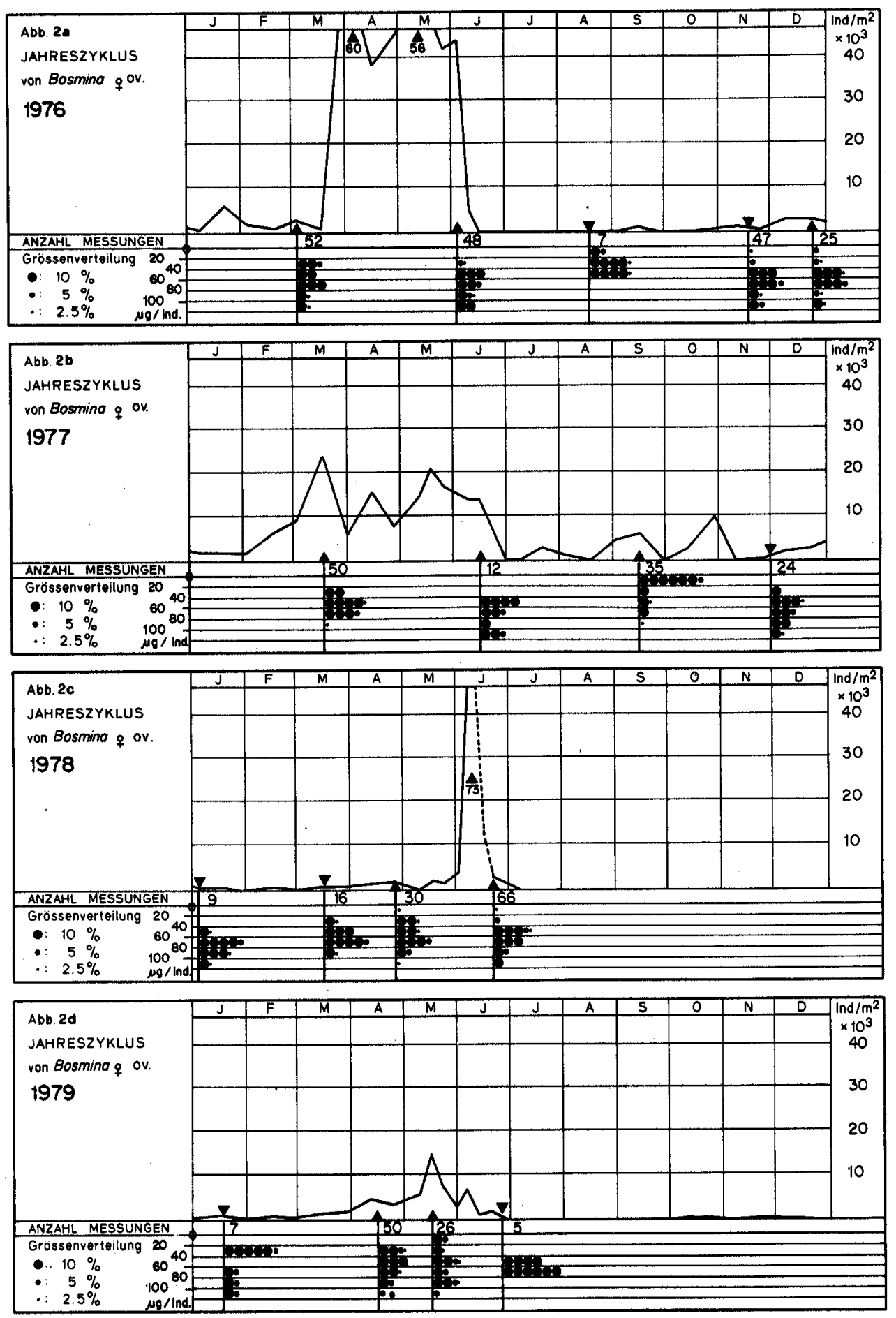

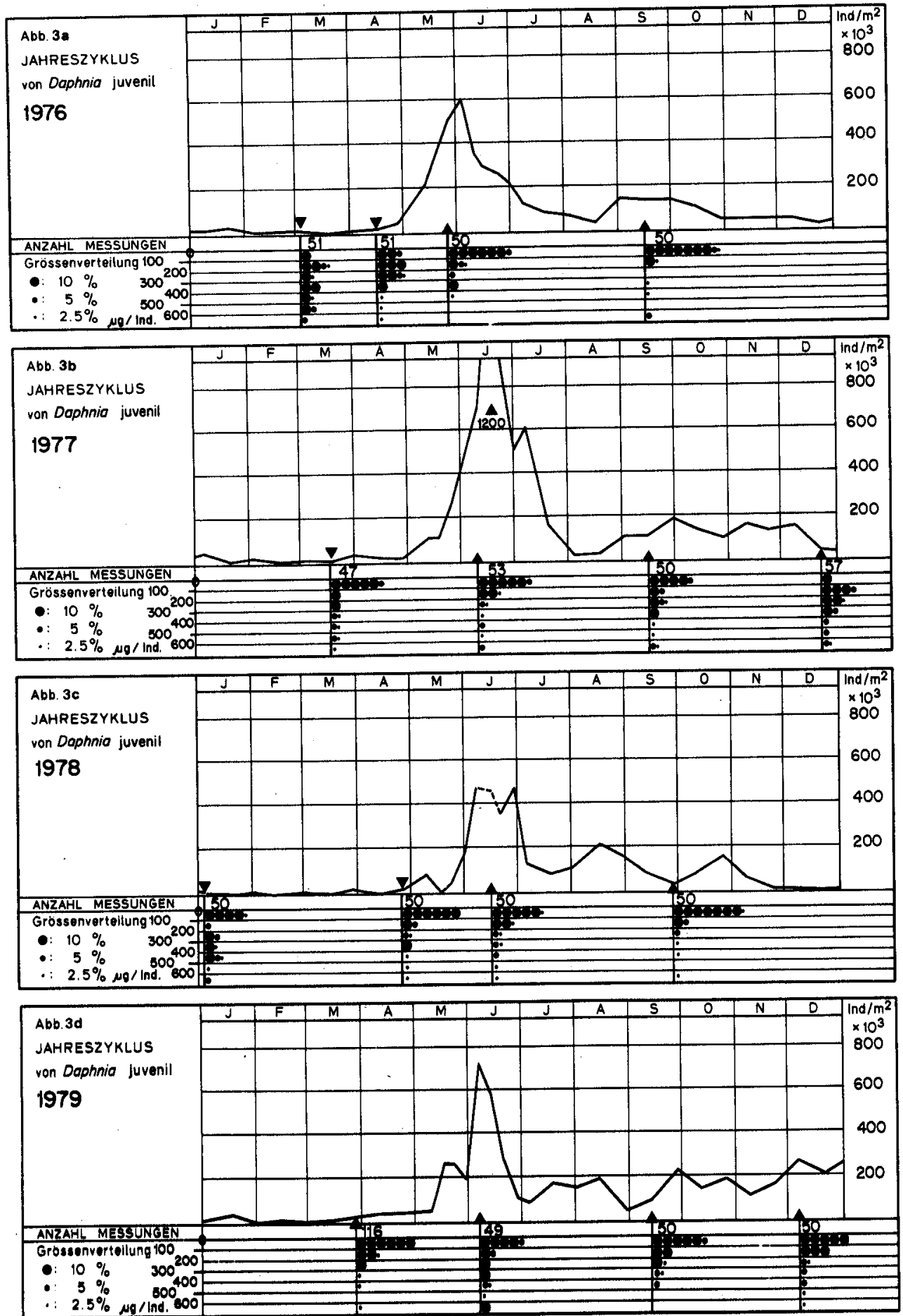

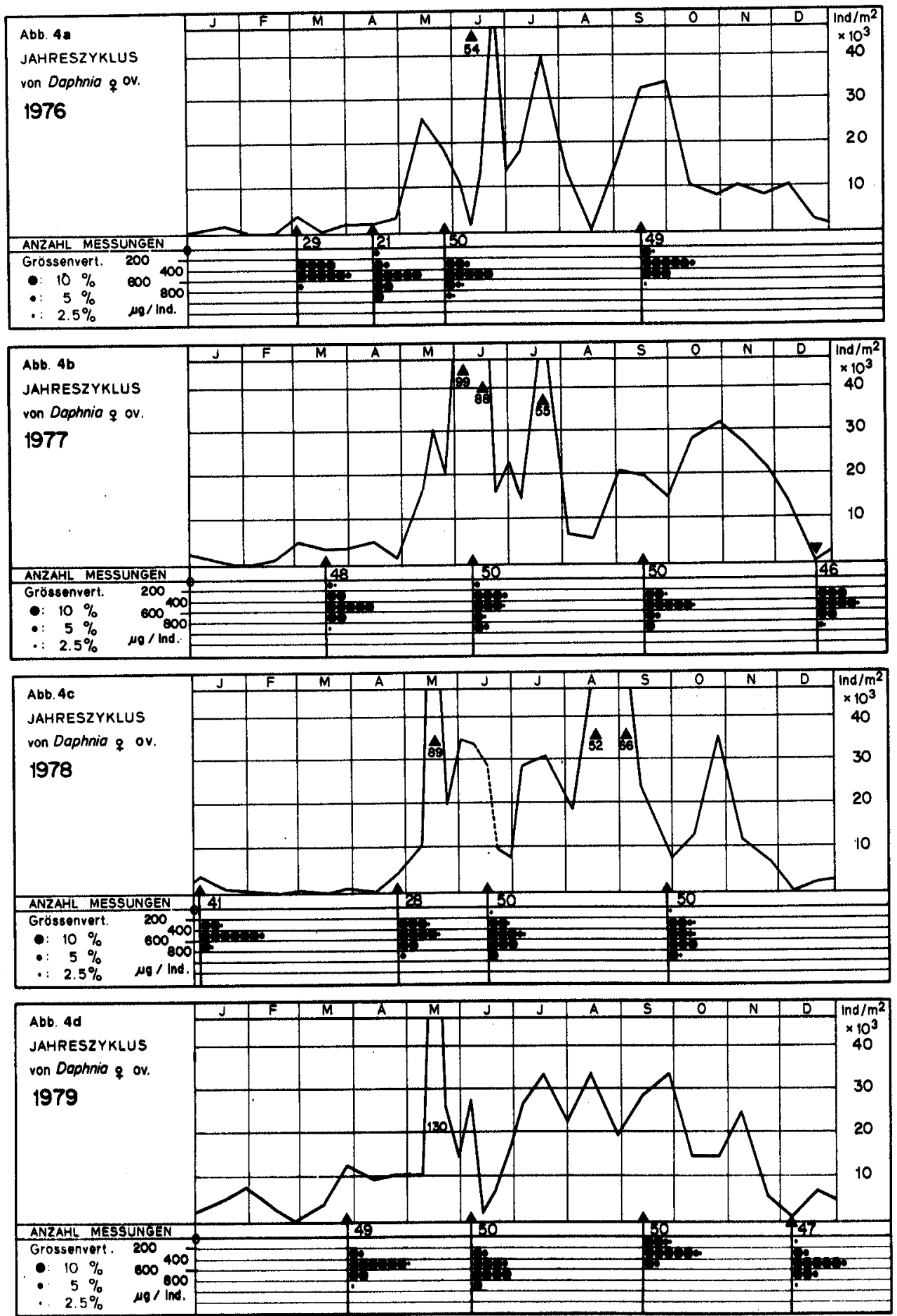

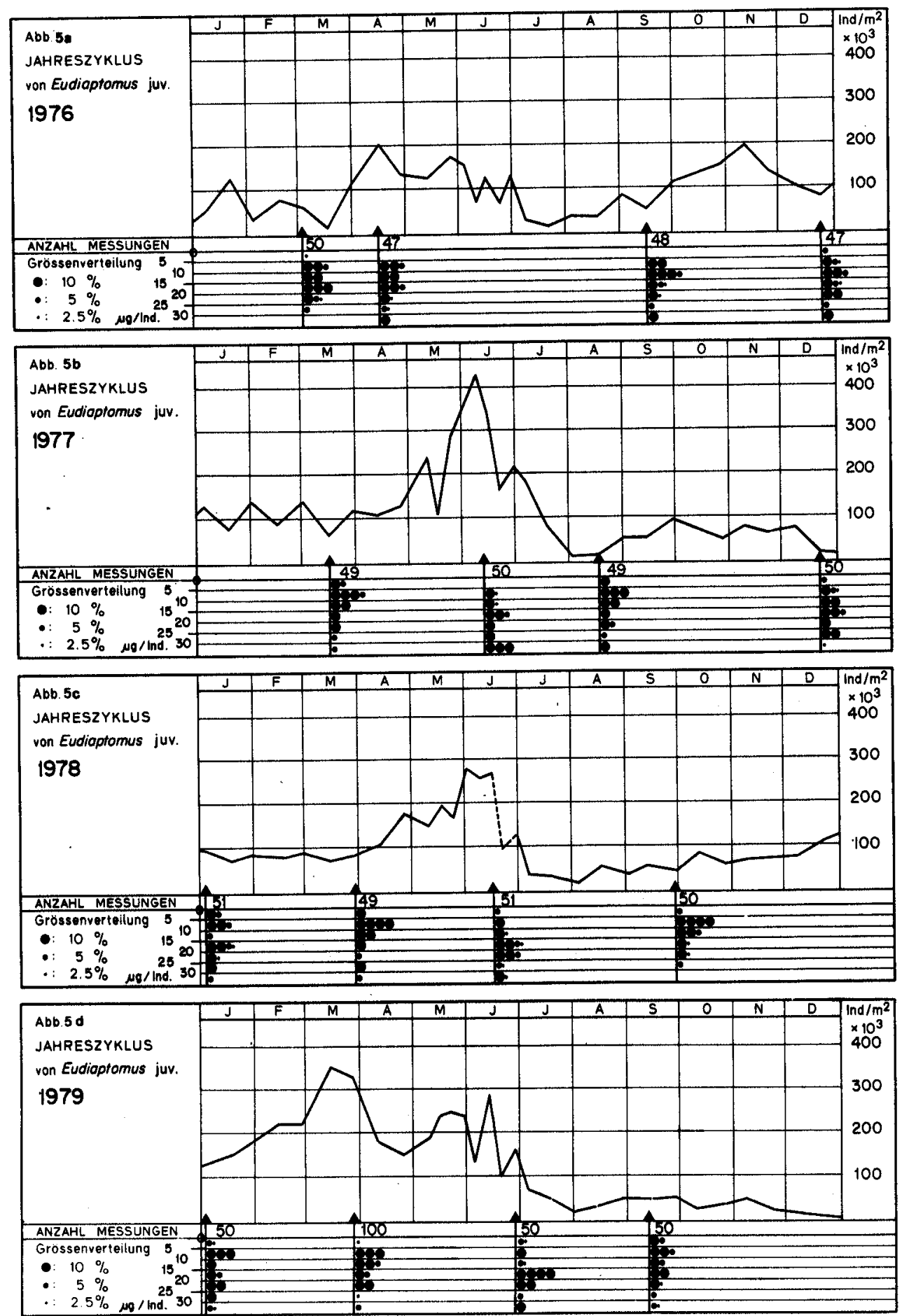

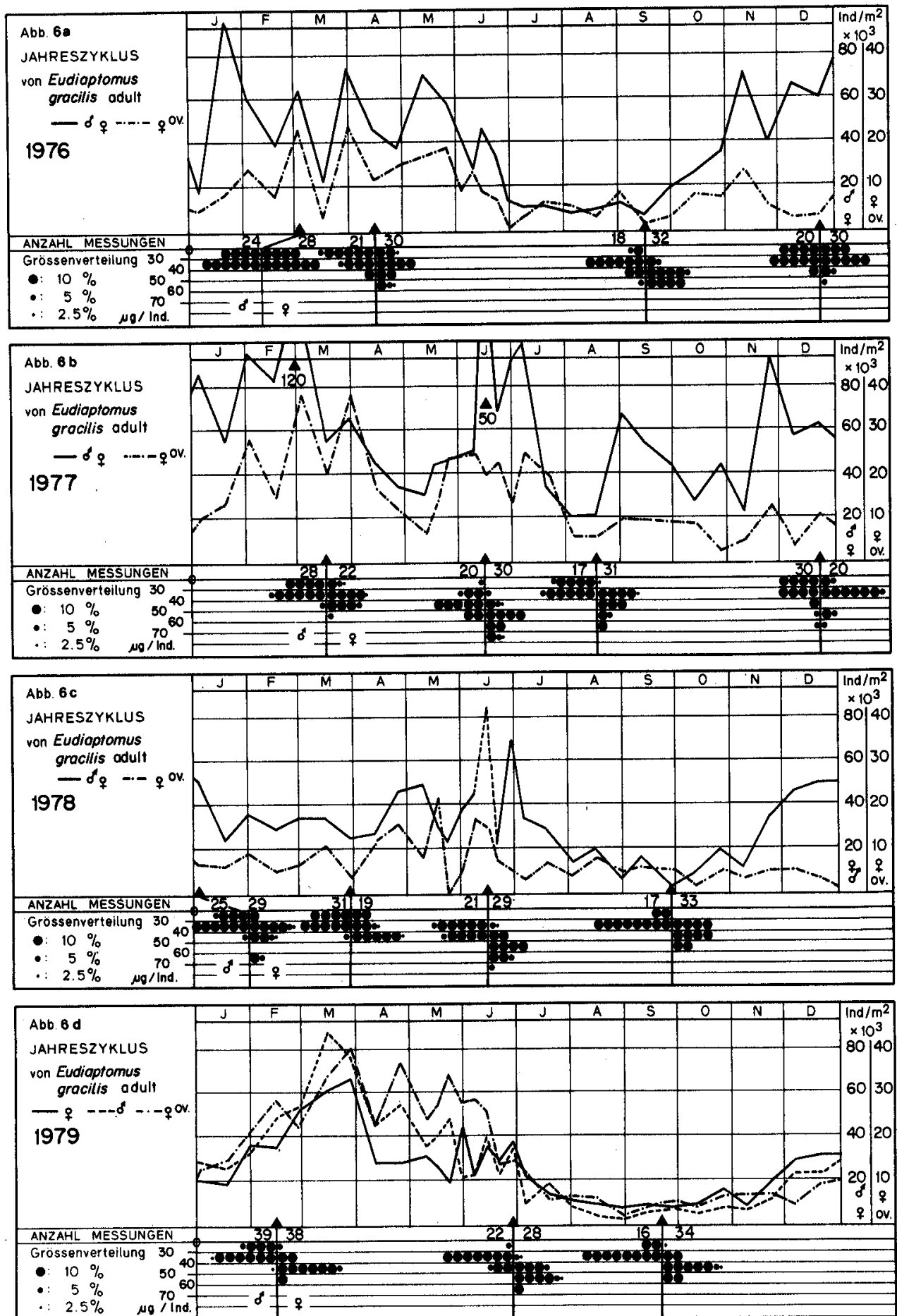

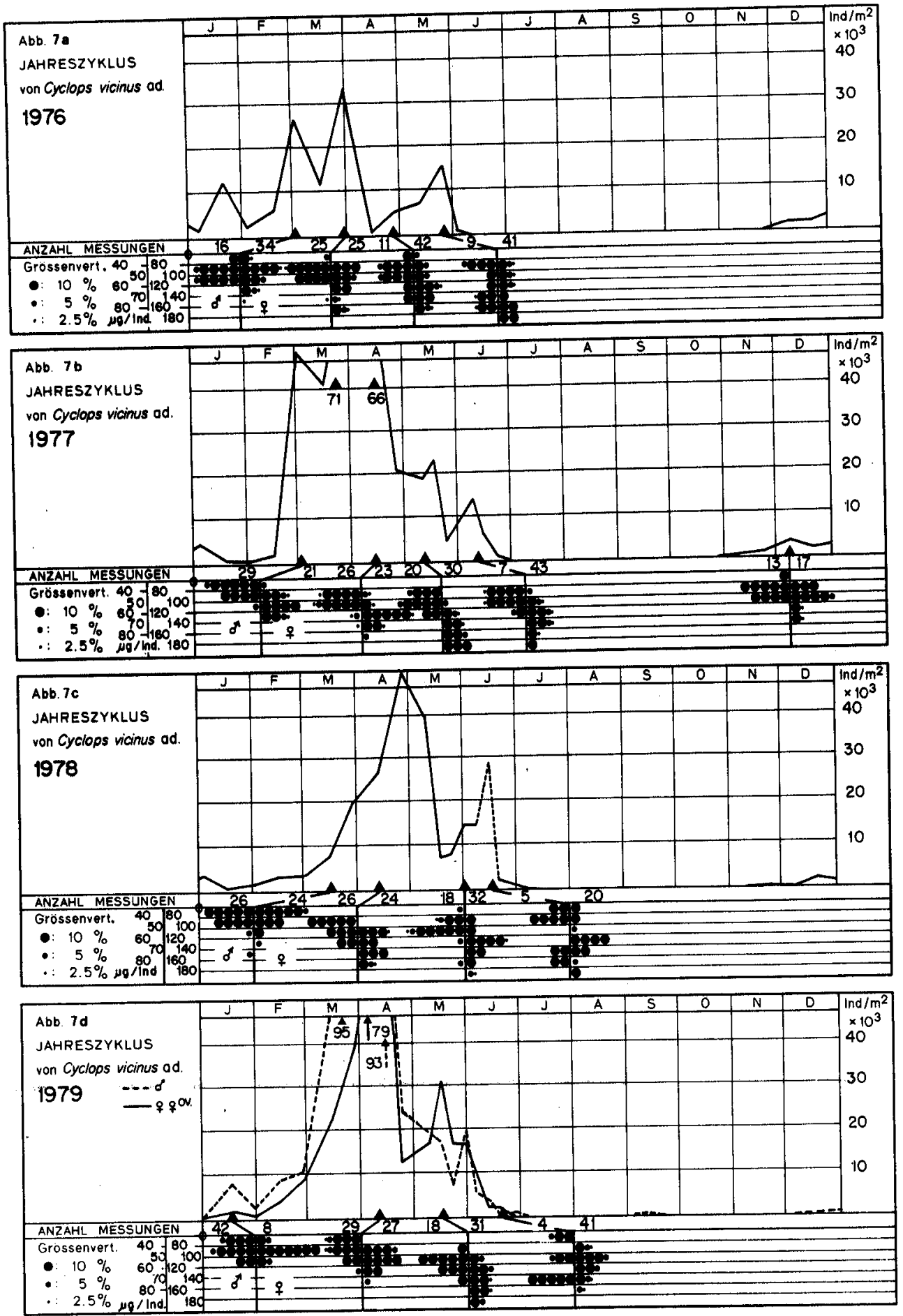

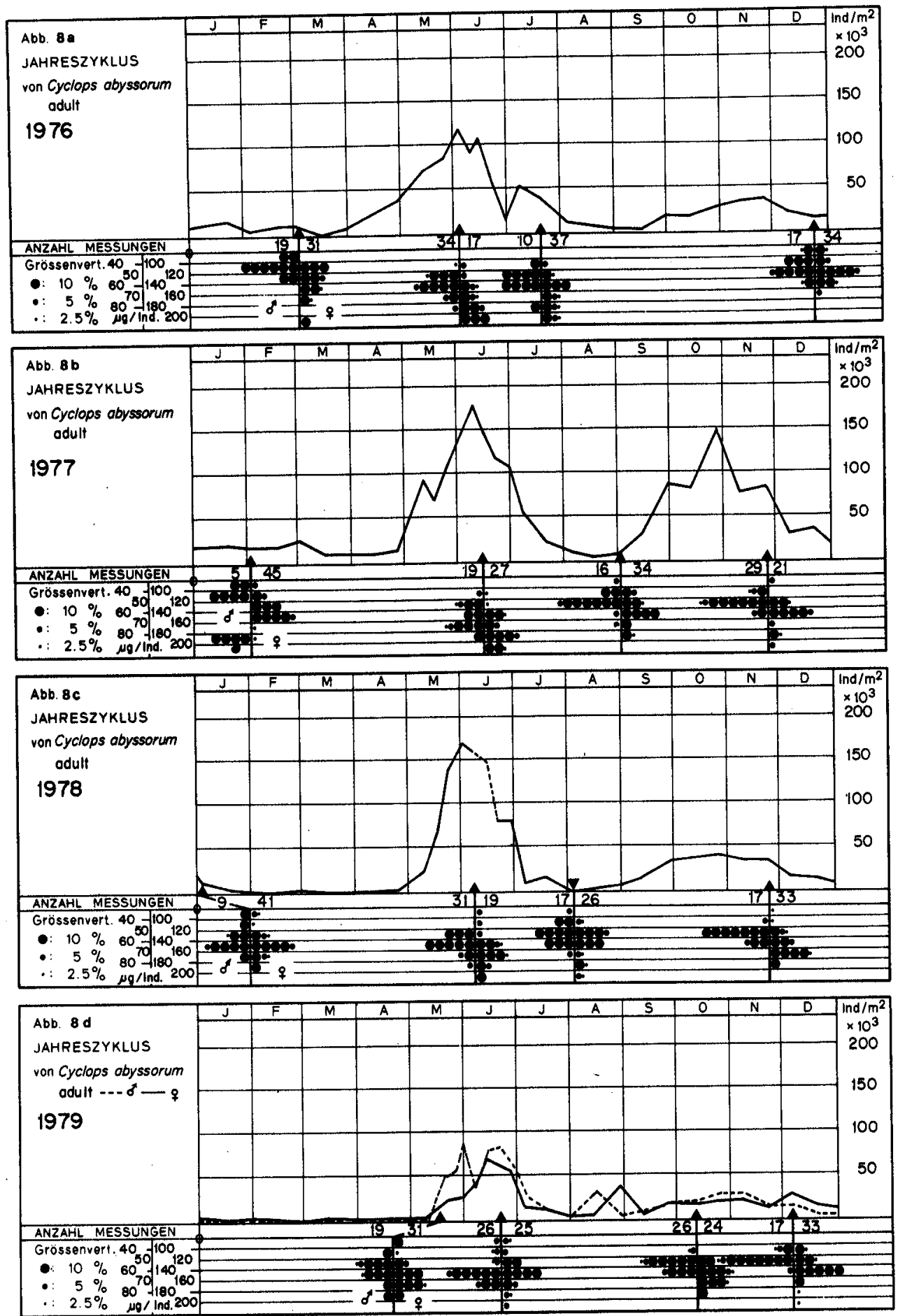

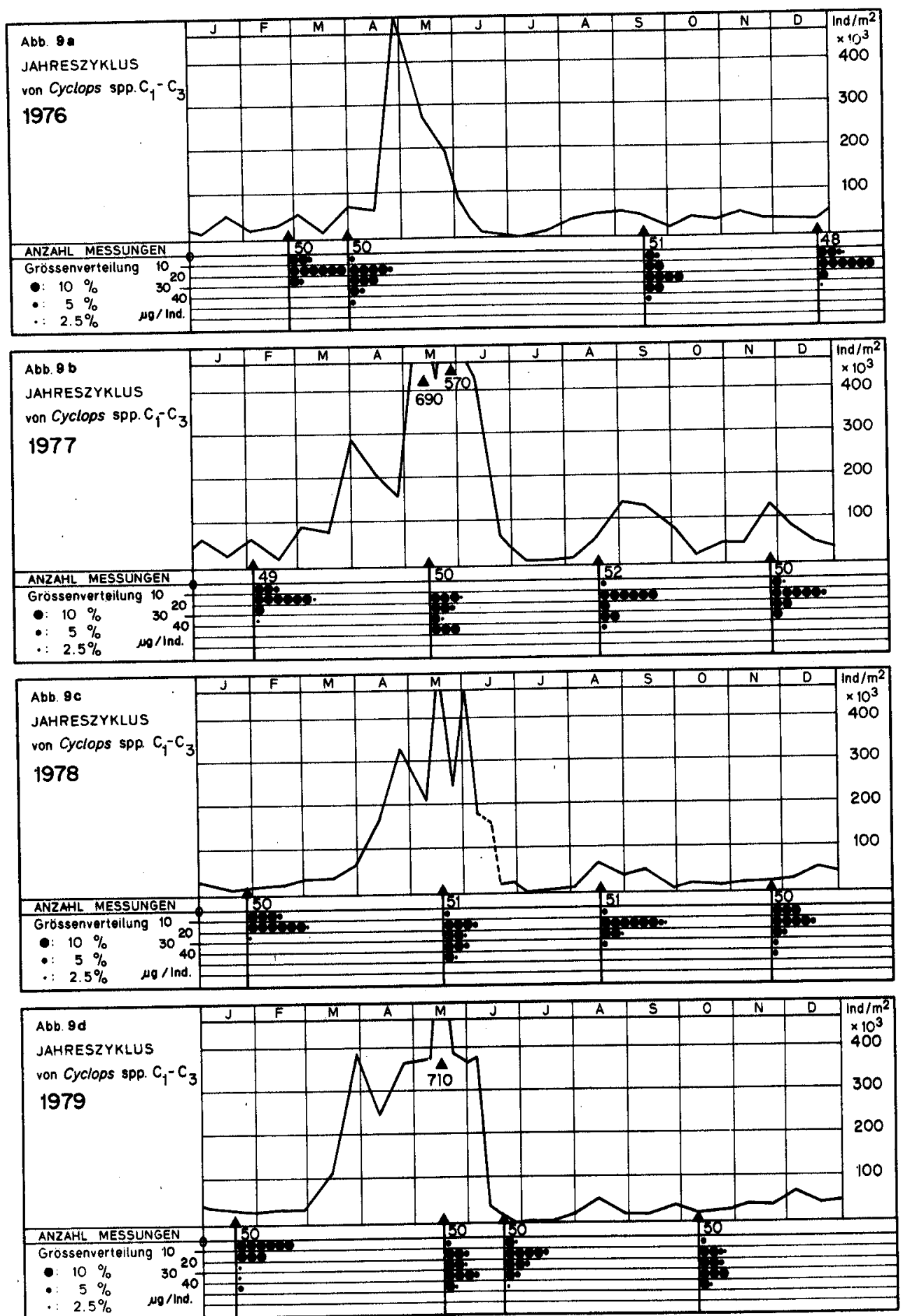

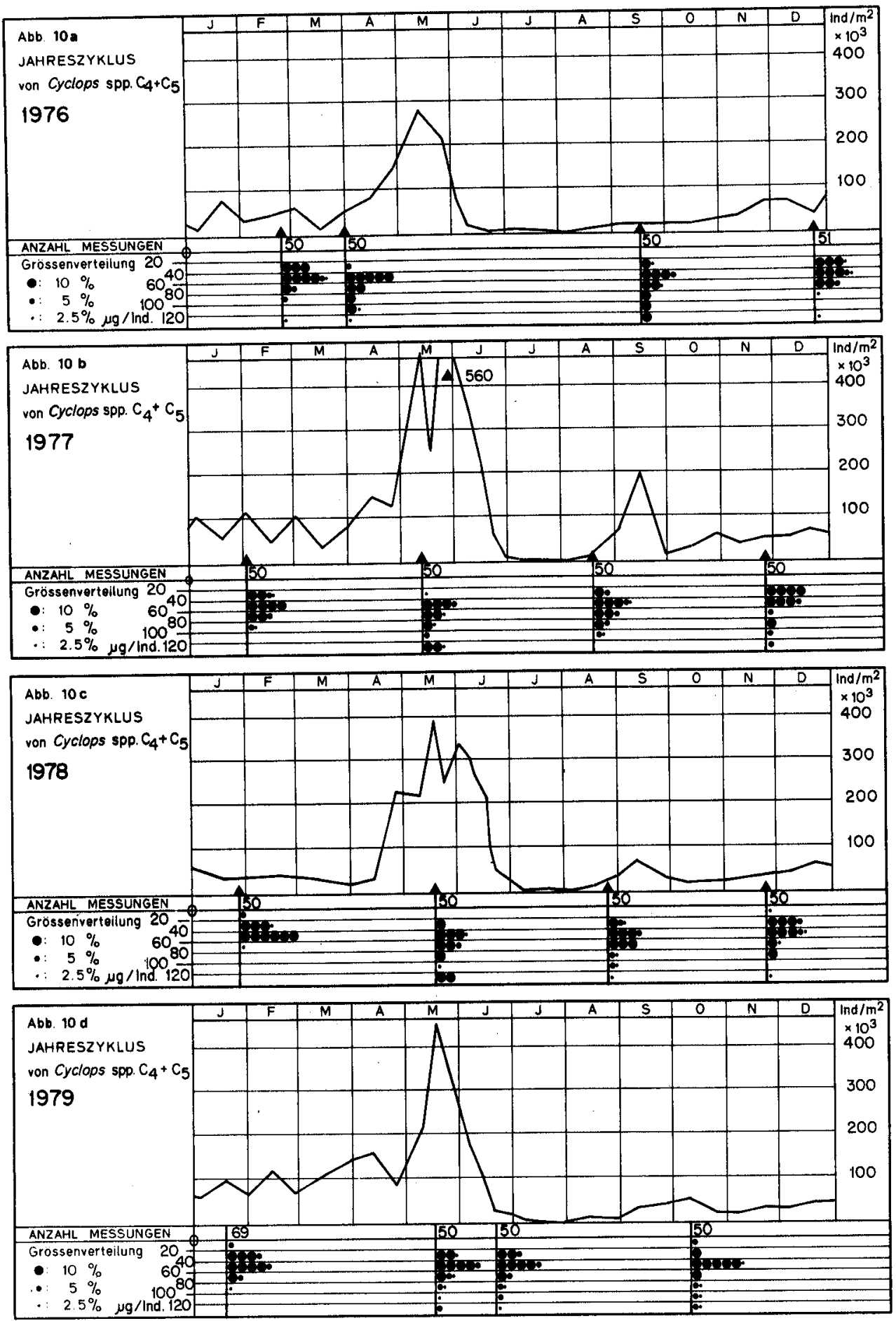

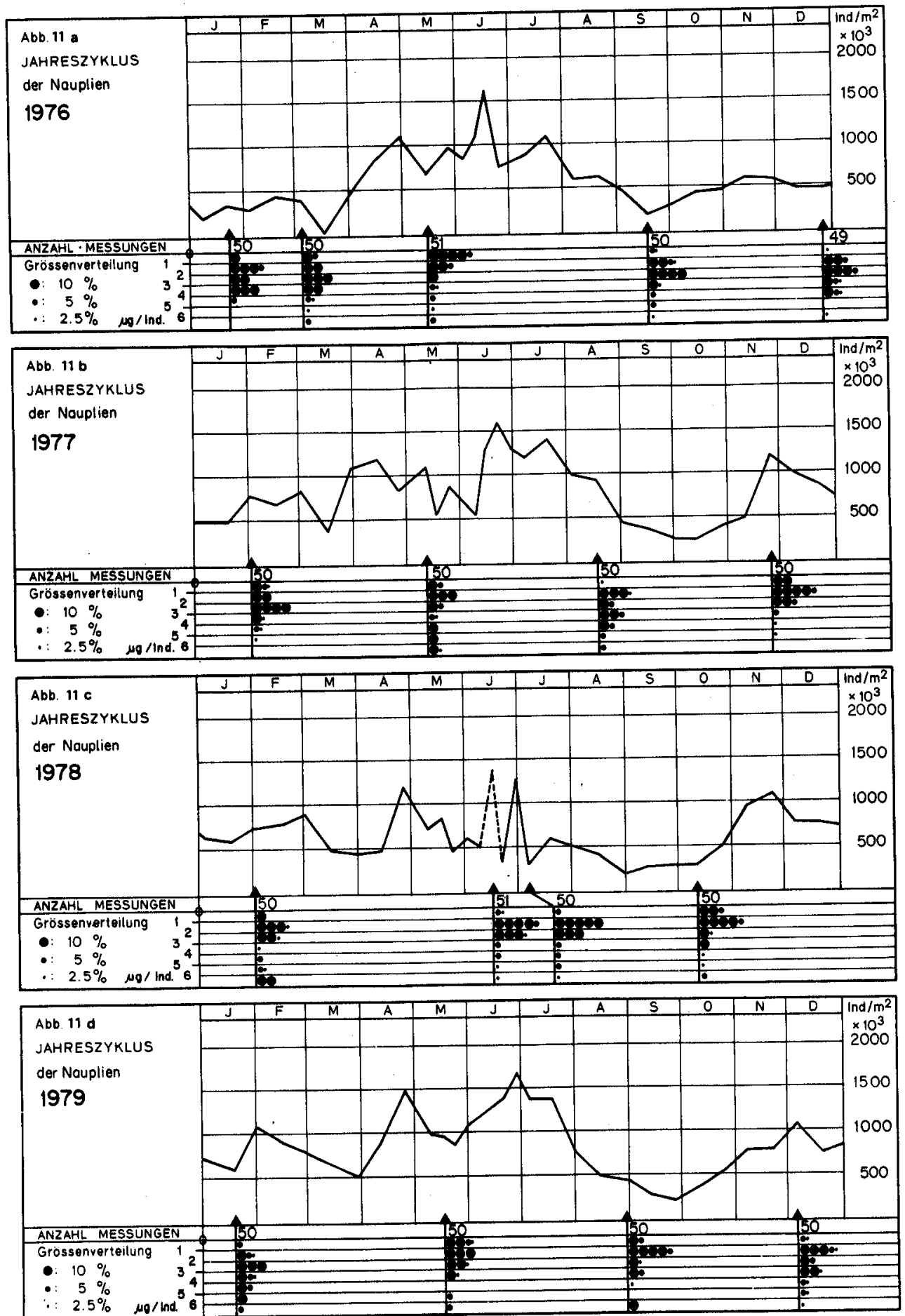

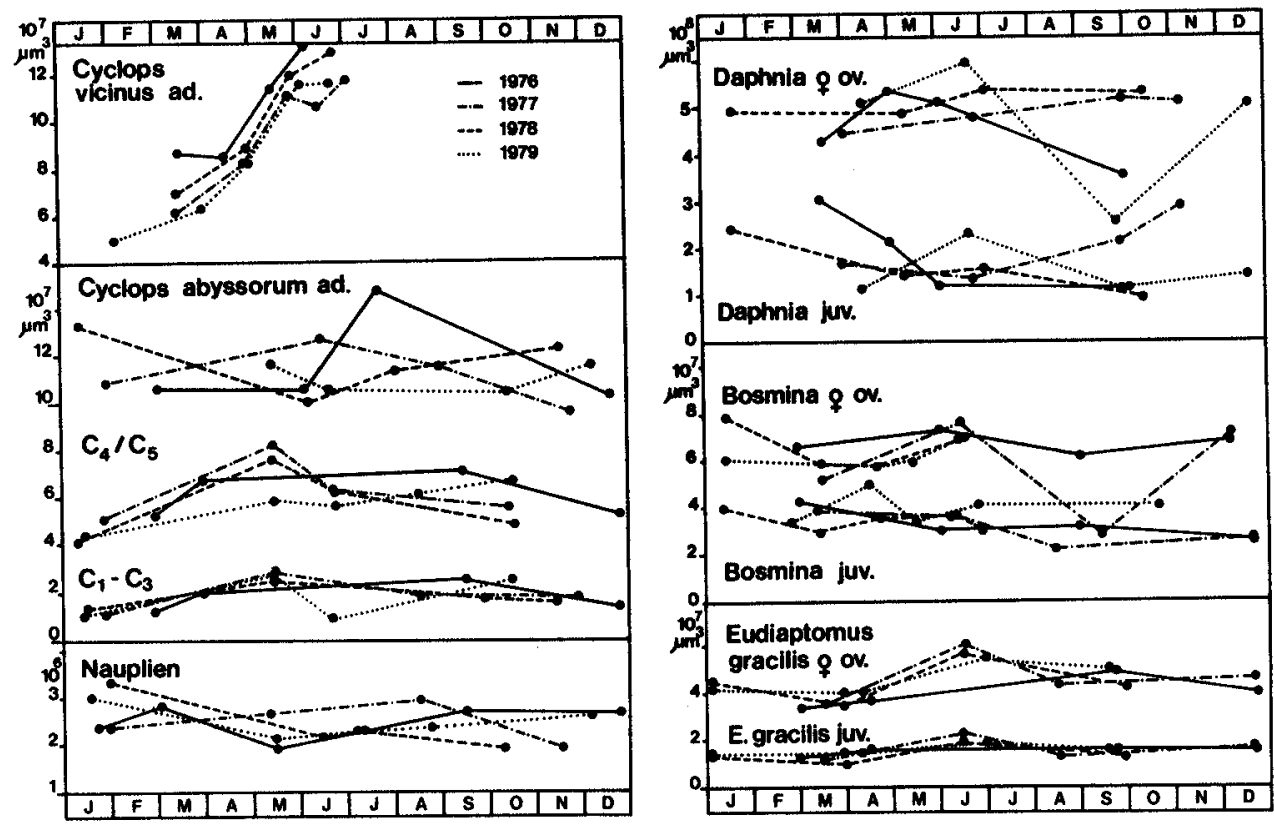

Abb. 12. Gemittelte Individuen-Volumina der häufigsten Crustaceen des Vierwaldstättersee-Planktons im Jahresverlauf.

Figure 12. Mean individual volumes of the most abundant crustaceans in Lake Lucerne plankton over the year.

wicklungsgänge zeigen eine Depression in der zweiten Jahreshälfte, wobei deren Dauer von Jahr zu Jahr variiert. Die Entwicklungsphasen der Juvenilstadien und der eiertragenden Weibchen sind direkt vergleichbar, wobei sie allerdings um rund 2 Wochen phasenverschoben sind. Die getrennte Analyse der $q$, $q$ ov und $\delta$ (Abb. 6d) zeigt parallele Entwicklungsgänge, wobei üblicherweise rund $35 \%$ aller Weibchen Eier trugen. Der Anteil der Männchen sinkt im April/Mai auf 30\% des Gesamtbestandes der adulten Tiere ab, steigt dann aber wieder auf ca. $40 \%$ an. Die Volumina (Abb. 12) schwanken im Jahresverlauf nur geringfügig. Einzig die Juni- und Juli-Werte erhöhen sich bei juvenilen und adulten Tieren um etwa $40 \%$ gegenüber dem April-Wert. Im August liegt das Volumen schon wieder beim langfristigen Mittel.

Die Männchen sind stets 20 bis 30\% kleiner (Volumen) als die Weibchen (Abb.6). Zwischen den eiertragenden und den übrigen adulten Weibchen besteht kein Grössenunterschied. Die Grössenverteilung der juvenilen Eudiaptomus gibt mehrfach Hinweise darauf, dass oft 2 Generationen gleichzeitig vorliegen.

\subsection{Cyclops vicinus (Abb.7)}

Die Entwicklung von Cyclops vicinus verläuft monozyklisch. Dadurch, dass im 4. Copepodid-Stadium eine ausgedehnte Diapause eintritt, welche im Sediment zugebracht wird, verschwindet die räuberische Cyclops-Art ausgerechnet dann, wenn ihre poten- 
tiellen Beutetiere die grösste Abundanz erreichen. Dieses Verhalten ist offensichtlich streng mit der Tageslänge gekoppelt und verändert sich nicht bei Zunahme des Nahrungsangebotes. Schon seit seinem ersten Auftreten im Vierwaldstättersee in den frühen 60er Jahren [4] zeigt diese Art dieselbe Jahresdynamik.

Die monozyklische Entwicklung spiegelt sich auch in der Grössenverteilung wider: Die Biomasse der adulten Cyclops-Individuen nimmt nach dem ersten massiven Auftreten im März bis zum Zusammenbruch der Adultgeneration im Mai/Juni deutlich zu, und zwar um rund 100\% (Abb. 12). Die linearen Abmessungen sind - bei gleichbleibenden Körperproportionen - bei den Männchen rund 25\% geringer als bei den Weibchen, was sich in einem Unterschied der Körpervolumina von 1:2 auswirkt. Die Bestandesdichten von Männchen und Weibchen sind ausgeglichen (vergl. Abb. 7d). Die Maxima der Weibchen folgen denjenigen der Männchen mit 3 Wochen Verzögerung.

\subsection{Cyclops abyssorum (Abb.8)}

Diese Form wurde von Mittelholzer [6] als typische Spätsommer/Herbstform des Vierwaldstättersees beschrieben. Heute erreicht sie ihre grösste Dichte im Mai/Juni; es lassen sich 2 Generationenfolgen von Adulten erkennen, welche aber drei CopepodidenGenerationen entspricht (Abb.9, 10). Diese Diskrepanz beruht z. T. auf rein zähltechnischen Unzulänglichkeiten, weil bei Routine-Untersuchungen die Zuordnung der Jugendstadien der Copepoden zu den Arten nicht möglich ist. In den Abbildungen 9 und 10 sind daher die Copepodiden der vorhandenen Cyclops arten kumuliert.

Die Entwicklung der Körpergrösse unterliegt bei C.abyssorum keiner sichtbaren Gesetzmässigkeit; die Mittelwerte aller Untersuchungen halten sich stets auf dem gleichen Niveau (Abb. 12). Auch bei dieser Form sind die Weibchen rund doppelt so gross wie die Männchen. Diese erreichen (1979) ungefähr die gleiche Populationsdichte, erscheinen aber 2 bis 3 Wochen vor den Weibchen.

\subsection{Nauplien und Copepodide (Abb.9, 10, 11)}

Die Jugendformen der Copepoden konnten aus zeitlichen Gründen nicht in die einzelnen Entwicklungsstadien aufgegliedert werden. Unter "Nauplien» sind alle Erststadien der Diaptomiden und Cyclopiden zusammengefasst; die Kategorien $C_{1}$ bis $C_{3}$ und $\mathrm{C}_{4}+\mathrm{C}_{5}$ schliessen die Copepodiden aller Cyclops arten ein. Trotz dieser Inhomogenität der Zählkategorien (oder gerade deswegen) zeigen die Grössenfraktionen geringe zeitliche Variabilität. Nur bei den Copepodiden tritt im Mai ein Maximum auf. Durch die zeitlich gestaffelten Eischübe der verschiedenen Copepoden wird die Jahresdynamik der Nauplien unstet. Bei den Copepodiden wiederum ist die Entwicklung des C.vicinus bis zum $\mathrm{C}_{4}$-Stadium mit $C$. abyssorum, welcher simultan die Entwicklung zur Adultgeneration vollzieht, überlagert. Die Grössenverteilung der $C_{4}$ und $C_{5}$ im Herbst zeigt, dass die Novembergeneration als Nachfahrin der Augustgeneration zu werten ist, da sie vermehrt kleinere Individuen aufweist.

\subsection{Weitere Crustaceen des Vierwaldstättersee-Pelagials (Tab. 1)}

Die selteneren, sporadisch bis regelmässig auftretenden Crustaceen-Arten sind in der Tabelle 1 aufgelistet. $\mathrm{Zu}$ den regelmässig und periodisch erscheinenden Arten gehören Leptodora kindtii und Bythotrephes longimanus. Sie prägen das Planktonbild im Hoch- 
sommer. Infolge der geringen Individuendichte lassen sich für die sporadisch auftretenden Cyclops bohater, Megacyclops gigas (Bodenform) und Mixodiaptomus laciniatus keine sicheren zeitlichen Präferenzen angeben; die adulten Tiere erscheinen eher in der zweiten Jahreshälfte. Einigermassen regelmässig erscheint der kleine Copepode Mesocyclops leuckarti, wobei sich die Maxima mit ca. $2400 \mathrm{Ind} / \mathrm{m}^{2} \not \hat{\sigma}+$ ad. im Vergleich mit dem weiter vorne beschriebenen Cyclops vicinus und Cyclops abyssorum bescheiden ausnehmen. Wie weit Diaphanosoma brachyurum zum festen Bestand des Vierwaldstätterseeplanktons gehört, ist noch ungewiss.

\section{Die Veränderungen des Crustaceenplanktons im Vergleich zu früheren Bestandesaufnahmen im Vierwaldstättersee}

Neben den eingehenden, 10 Jahre früher erfolgten populationsdynamischen Untersuchungen von Mittelholzer [6] im Gersauerbecken des Vierwaldstättersees und den Untersuchungen von Burckhardt [1] aus 1898 sind nur punktuelle, nicht quantifizierbare Analysen vorhanden $[2,11]$.

Dank der Bestimmung des Filtrationskoeffizienten mit Hilfe eines Vergleichs der Pumpfänge mit der Netzmethode, lassen sich die von Burckhardt erfassten Individuendichten einigermassen quantifizieren. Als Vergleichsbasis sind die jeweils beobachteten Maxima in der Tabelle 2 zusammengefasst.

Mit Ausnahme von Mesocyclops leuckarti und Mixodiaptomus laciniatus haben alle Crustaceen im Laufe der Zeit deutlich zugenommen. Ins Auge springt besonders die Entwicklung der herbivoren Cladoceren (Daphnia- und Bosmina-Arten) und des räuberischen Cyclops vicinus. Seit den 10 Jahre zurückliegenden Zählungen von Mittelholzer sind diese Formen auf das Zehnfache angestiegen. Allerdings sind bei diesem Vergleich die Verbesserungen der Fangtechnik (effizientere Netze) ebenso zu berücksichtigen wie die Tatsache, dass Mittelholzer seine Netzzüge im Gersauerbecken des Vierwaldstättersees erhob. Wie ein nachträglicher Quervergleich von 6 Seebecken des Vierwaldstättersees ergab, weist diese Stelle - trotz ihrer grossen Tiefe von $214 \mathrm{~m}$ - geringere flächenspezifische Individuendichten auf, als der $111 \mathrm{~m}$ tiefe Kreuztrichter.

Selbst bei grosszügiger Interpretation dieser methodischen Unstimmigkeiten bleiben jedoch Zuwachsraten von 50 bis $500 \%$ je nach Art erhalten. Auch die relativen Verschiebungen der Häufigkeiten der einzelnen Arten bleiben nach der Korrektur durch die Befunde des Quervergleichs der Probenahmestellen bestehen.

Die überproportionale Zunahme der herbivoren Cladoceren kann mit der Veränderung der Nahrungsbasis erklärt werden. Als Folge der Eutrophierung erreichten die schnellwüchsigen Nanoplankter (wichtigste Futterquelle für herbivore Crustaceen) höhere Biomasse-Anteile: 5,5 g Nassgewicht/ $\mathrm{m}^{2}$ (langjähriges Mittel 1975-1980) gegenüber 3.1 $\mathrm{g} / \mathrm{m}^{2}$ im Jahre 1968.

Der Gesamtphosphor-Gehalt zur Zeit der Vollzirkulation kann als Gradmesser der Eutrophierung dienen. Dieser Wert lag zur Zeit der Untersuchungen von Mittelholzer [6] in der trophogenen Schicht $(0-20 \mathrm{~m})$ noch bei 19 bis $21 \mathrm{mg} / \mathrm{m}^{3}$ gegenüber dem höchsten Wert von $32 \mathrm{mg} / \mathrm{m}^{3}$ im Jahre 1976. Klimatische Ausnahme-Situationen (z. B. extrem warme Trockenjahre oder milde Winter) wirkten sich über unvollständige Zirkulation auf den Sauerstoff-Haushalt und die Folgereaktionen aus. Die biologische 
Tabelle 1: Seltenere Crustaceen im Vierwaldstättersee

\begin{tabular}{|c|c|c|c|}
\hline Art & $\begin{array}{l}\text { berechnetes } \\
\text { Körpervolumen } 10^{6} \mu^{3}\end{array}$ & $\begin{array}{l}\text { Periode mit } \\
\text { gehäuftem Vorkommen } \\
\text { adulter Tiere }\end{array}$ & $\begin{array}{l}\text { grösstes beobachtetes } \\
\text { Maximum adulter } \\
\text { Tiere [Ind/ } \mathrm{m}^{2} \text { ] }\end{array}$ \\
\hline $\begin{array}{l}\text { Mesocyclops } \\
\text { leuckarti }\end{array}$ & $\begin{array}{l}\delta 10.6 \pm 2 \\
\text { 앙 ov. } 35 \pm 5.6\end{array}$ & Aug/Sept & 2400 \\
\hline $\begin{array}{l}\text { Cyclops } \\
\text { bohater }\end{array}$ & $\sigma^{*}, q, q$ ov. $430 \pm 210$ & Okt-Dez & 1400 \\
\hline $\begin{array}{l}\text { Megacyclops } \\
\text { gigas }\end{array}$ & $\begin{array}{l}\text { đ } 490 \pm 80 \\
\text { q, } q \text { ov. } 1370 \pm 410\end{array}$ & Mai-Juli & 330 \\
\hline $\begin{array}{l}\text { Mixodiaptomus } \\
\text { laciniatus }\end{array}$ & $\begin{array}{l}\text { o } 66 \pm 18 \\
\text { 우 } 144 \pm 31 \\
\text { 아. } 165 \pm 32\end{array}$ & Mai-Juli & 1500 \\
\hline $\begin{array}{l}\text { Leptodora } \\
\text { kindtii }\end{array}$ & & Juli/Aug/Sept & 3600 \\
\hline $\begin{array}{l}\text { Bythotrephes } \\
\text { longimanus }\end{array}$ & & $\begin{array}{l}\text { Juni/Juli/ } \\
\text { Aug/Sept }\end{array}$ & 7300 \\
\hline $\begin{array}{l}\text { Diaphanosoma } \\
\text { brachyurum }\end{array}$ & & Okt/Nov & 1400 \\
\hline
\end{tabular}

Tabelle 2: Vergleich der gefundenen maximalen Individuendichten mit Resultaten früherer Untersuchungen von BURCKARDT und MITTELHOLZER

\begin{tabular}{|c|c|c|c|}
\hline Gattung/Art & Individuendichte $/ \mathrm{m}^{2}$ & & \\
\hline & $\begin{array}{l}\text { nach } \\
\text { BURCKHARDT*) } \\
1898 \\
\end{array}$ & $\begin{array}{l}\text { nach***) } \\
\text { MITTELHOLZER } \\
\text { 1967/68 }\end{array}$ & $\begin{array}{l}\text { nach } \\
\text { BUERGI u. EGLI } \\
\text { 1976-79 } \\
\end{array}$ \\
\hline $\begin{array}{l}\text { Eudiaptomus } \\
\text { gracilis ad. }\end{array}$ & 140000 & 53000 & 170000 \\
\hline $\begin{array}{l}\text { Mixodiaptomus } \\
\text { laciniatus ad. }\end{array}$ & 27000 & $\begin{array}{l}\text { keine } \\
\text { Angaben }\end{array}$ & 1500 \\
\hline $\begin{array}{l}\text { Mesocyclops } \\
\text { leuckarti ad. }\end{array}$ & 7600 & 9400 & 2400 \\
\hline Cyclops vicinus ad. & - & 140000 & 175000 \\
\hline C. strenuus**) & 32000 & & \\
\hline C. abyssorum & - & 55000 & 180000 \\
\hline Nauplien & 118000 & 800000 & 1600000 \\
\hline $\begin{array}{l}\text { Daphnia hyalina } \\
\text { juv. + ad. }\end{array}$ & 64000 & 114000 & 1289000 \\
\hline Bosmina juv. + ad. & - & 66000 & 514000 \\
\hline Leptodora kindtii & 1840 & $5(?)$ & 3600 \\
\hline $\begin{array}{l}\text { Bythotrephes } \\
\text { longimanus } \\
\end{array}$ & 320 & 4500 & 7300 \\
\hline
\end{tabular}

*) korrigiert mit Filtrationskoeffizient

**) Nach EINSLE U. handelt es sich hier um C. abyssorum (mündl. Mitteilung)

***) Stelle Gersauerbecken 
Reaktion setzte gemäss den Generationszeiten schrittweise verzögert ein: Die Primärproduzenten profitierten unmittelbar von der erhöhten Nährstoffzufuhr, die Protozoen und Rotatorien folgten mit erhöhter Biomasseproduktion schnell nach, während die Verschiebungen im Artengefüge der Crustaceen viel mehr Zeit benötigten und erst nach Jahren in ein neues Gleichgewicht führten.

\section{Zusammenfassung}

Während einer vierjährigen Untersuchung wurden im Vierwaldstättersee (Stelle Kreuztrichter) in Intervallen von ein bis zwei Wochen Zooplanktonproben quantitativ erhoben und gezählt. Um die Variabilität der Körpergrösse festzustellen, wurden die häufigeren Crustaceen in statistisch ausreichender Anzahl in ausgewählten Entwicklungsphasen ausgemessen.

Die Abfolge der verschiedenen Populationen weist auf mehrere steuernde Ökofaktoren hin:

- Mit dem ersten Temperatur-Anstieg im März wird eine progressive Entwicklung der herbivoren Cladoceren bzw. eine Weiterentwicklung der überwinternden Jugendstadien der Copepoden zur Adultgeneration ausgelöst.

- Günstige Nahrungsbedingungen kennzeichnen die Periode vom April bis Mai sowohl für herbivore Filtrierer wie für Carnivore. Bis zum Zusammenbruch des Phytoplanktons im Juni nimmt die Individuendichte der herbivoren Cladoceren sprunghaft zu.

- Ohne Rücksicht auf die Nahrungsituation, welche zu diesem Zeitpunkt sehr vorteilhaft ist, zwingt das durch die Tageslänge ausgelöste Verhaltensmuster Cyclops vicinus im $\mathrm{C}_{4}$-Stadium zur Diapause im Seesediment. Seine Entwicklung ist monozyklisch. Zwei deutliche Generationen und Hinweise auf eine dritte Generation finden sich bei Cyclops abyssorum.

- Trotz weitgehendem Fehlen carnivorer Zooplankter kommt es im Juni/Juli infolge Nahrungsmangel zum markanten Rückgang der herbivoren Crustaceen, welcher bei den Phyllopoden mit der Bildung von Latenzeiern verbunden ist.

- Die grossen Raubcladoceren (Bythotrephes und Leptodora) verhindern während der Hochsommerzeit ein erneutes Ansteigen der filtrierenden Crustaceen, welche zusätzlich unter einem Mangel an geeigneten Futteralgen leiden, weil - nicht zuletzt durch die selektive Nahrungsauswahl bedingt - nur noch grosse, aber nicht verwertbare Algen übrigbleiben.

Im Vergleich mit früheren Planktonanalysen fällt eine Zunahme der herbivoren Crustaceen auf. Die Maxima von Daphnia und Bosmina haben sich in den 10 Jahren seit der letzten Untersuchung verfünffacht. Aber auch der räuberische Cyclops vicinus und weniger deutlich die Raubcladoceren konnten ihre Individuendichten steigern, während die früher schon seltenen Copepoden Mixodiaptomus laciniatus und Mesocyclops leukkarti weiter an Bedeutung verlieren.

Die Körpergrösse zeigt im Jahresverlauf - von wenigen Ausnahmen abgesehen - keine grosse Variabilität. Einzig in der Periode vom März bis Mai sind signifikante Änderungen der Körpergrösse feststellbar: Bei den adulten Tieren nimmt sie bis $\mathrm{zu} 100 \% \mathrm{zu}$. Die juvenilen Stadien machen diese Entwicklung nur schwach mit oder verlieren in dieser Zeit sogar an Volumen. 


\section{SUMMARY}

During a 4-year field campaign, quantitative zooplankton samples were taken from Lake of Lucerne (sampling site: Kreuztrichter) at intervals of one to two weeks. To determine size variability, the statistically most abundant crustacean populations were measured at selected of development.

Succession of the various populations is an indication of several controlling ecological factors:

- With the first temperature increase in March, the herbivorous cladocerens undergo a progressive development, and the juvenile hibernating copepodes continue their growth to adulthood.

- April till May is a characteristic period for food abundance not only for the herbivorous filter feeders but also for carnivores. A marked increase in the density of the herbivorous cladocere population is observed in the dissappearance of phytoplankton in June.

- Cyclops vicinus whose diapause behavior pattern is steered by daylength, is forced in the $\mathrm{C}_{4}$ stage to retire to the lake sediment regardless of good conditions which are very favorable at this time of year. Its development is monocyclic. Two distinct generations and indications of a third one were observed with Cyclops abyssorum.

- Although carnivorous zooplankton populations are low, a significant reduction of the herbivorous crustaceans was observed in June/July as a consequence of food shortage, this corresponds to the period when phyllopodes form dormant eggs.

- During midsummer, the large cladoceren predators (Bythotrephes and Leptodora) prevent a renewed increase of crustacean filter feeders. During this period, the latter suffer additionally under acute food shortage due to their selective feeding on small algae, only large, unconsumable algae are left.

Compared to former plankton analysis, an increase of the herbivorous crustacean population is noted. Daphnia and Bosmina maxima quintupled in the past 10 years since the last analysis was carried out. The number of predatory Cyclops vicinus and less evidently the cladoceren predators also increased in density, while the formerly already rare copepode species Mixodiaptomus laciniatus and Mesocyclops leuckarti continue to decrease. Apart from a few exceptions, no great size variability was noted over the year. Only from March to May were significant size alterations noted: adult animals increased up to $100 \%$ in volume, while animals in juvenile stages increased only slightly or even decreased in volume during this period.

\section{RÉSUMÉ}

Pendant quatre ans, des échantillons de zooplancton ont été prélevés dans le lac des Quatre-Cantons (Kreuztrichter), et mesurés à intervalles d'une à deux semaines. Afin de déterminer la variabilité des tailles, les populations de crustacés les plus abondantes furent mesurées dans des phases de développement sélectionnés.

La succession des différentes populations révèle plusieurs facteurs écologiques déterminants:

- Avec la première augmentation de température en mars, les cladocères herbivores se développent progressivement, et les jeunes copépodes hibernant continuent leur developpement.

- Les conditions alimentaires caractéristiques pour la période d'avril à mai sont propices non seulement pour les filtreurs herbivores mais aussi pour les carnivores. Jusqu'à disparition du phytoplancton en juin, le nombre de cladocères herbivores augmente intensivement en densité.

- Sans tenir compte des conditions alimentaires qui sont très propices à cette époque, Cyclops vicinus dans la phase $\mathrm{C}_{4}$ qui base son comportement sur la durée de luminosité, est forcé à la diapause dans le sédiment $\mathrm{du}$ lac. Son développement est monocyclique. Deux générations évidentes et des indications d'une troisième ont pu être observées chez Cyclops abyssorum.

- Pendant une période où les populations de zooplancton carnivores sont largement absentes, une diminution marquante des crustacés herbivores fut observée en juin/juillet en raison du manque de nourriture qui correspond à la période où les phyllopodes forment les œufs de résistance.

- En plein été, les grands cladocères prédateurs empêchent une nouvelle augmentation des crustacés filtreurs. A cette époque, ceux-ci ont de la peine à trouver assez de nourriture algale appropriée car, à part qu'ils soient limités par leur consommation selective de nourriture, il ne reste que des grandes algues inconsommables.

Comparé aux analyses antérieures de plancton, une augmentation des crustacés herbivores a été observée. Le nombre maximal de Daphnia et Bosmina a quintuplé depuis la dernière analyse d'il y a 10 ans. Le nombre de 
Cyclops vicinus prédateur a également augmenté en densité, et de manière moins évidente celui des cladocères prédateurs, tandis que les espèces déjà très rares de copépodes Mixodiaptomus laciniatus et Mesocyclops leuckarti perdent de plus en plus d'importance.

Outre quelques exceptions, les tailles n'ont pas varié de manière importante au cours d'une année. Uniquement durant la période de mars à mai où des modifications appréciables des tailles ont pu être constatées. Durant cette période, la taille des animaux adultes augmente jusqu'à $100 \%$, tandis que les jeunes animaux se développent que très lentement ou diminuent même en volume.

\section{VERDANKUNG}

Für zahlreiche Ergänzungen des Manuskripts und die Hilfe bei den Bestimmungen der Crustaceen möchten wir Dr. U. Einsle unseren besten Dank aussprechen. Grossen Dank schulden wir Herrn Dr. U. Lemmin. Er besorgte in zuvorkommender Weise die EDV-Bearbeitung der Grössenklassen. Herrn Prof. H. Ambühl danken wir herzlich für die kritische Durchsicht und die stilistische Überarbeitung des Manuskripts. Für Résumé und Summary stand uns Frl. S. Peter hilfreich zur Seite.

\section{LITERATUR}

1 Burckardt, G.: Quantitative Studien über das Zooplankton des Vierwaldstättersees, Mitt. Naturf. Ges. Luzern 3, 192-439, 1900.

2 Burckardt, G.: Der Jahrescyclus der planktischen Entomostraken; nach Beobachtungen im Vierwaldstättersee und in den Aroser Hochgebirgseen. Festschrift für Zschokke 13, 23 p 1920.

3 Bürgi, H.R.: Eine neue Netzgarnitur mit Kipp-Schliessmechanismus für quantitative Zooplanktonfänge in Seen. Schweiz. Z. Hydrol. 45/2, 505-507, 1983.

4 Kiefer, F. und Einsle, U., Uber das Vorkommen verschiedener Arten der Gattung Cyclops (s. restr.) in schweizerischen Seen. Schweiz. Z. Hydrol. 24, 44-48, 1962.

5 Nauwerck, A., Die Biomasse; Gewichtsbestimmung, Symb. Bot. Upsal. 17/5, 1963.

6 Mittelholzer, E., Populationsdynamik und Produktion des Zooplanktons im Greifensee und im Vierwaldstättersee. Schweiz. Z. Hydrol. 32/1, 90-149, 1970.

7 Rott, E., Some results from phytoplankton counting intercalibrations. Schweiz. Z. Hydrol. 43/1, 34-62, 1981.

8 Ruttner-Kolisko, A., Suggestions for biomass calculation of plankton rotifers. Arch. Hydrobiol. Beih. Ergebn. Limnol. 8, 71-76, 1977.

9 Schlott-Idl. K., Populationsdynamik pelagischer Protozoen des Piburgersees (Tirol, Österreich). Diss. Abt. Limnol. 14: 1-82, Univ. Innsbruck.

10 Sebestyén, Olga, Quantitative Plankton Studies on Lake Balaton Ann. Inst. Biologici (Tihany) Hung. Acad. Scient. 25, 281-292, 1958.

11 Strickler, R., Die neuere Entwicklung des Zooplanktons im Vierwaldstättersee, Diplomarbeit ETHZ, 1965.

12 Utermöhl, H., Zur Vervollkommnung der quantitativen Phytoplankton-Methodik Mitt. Int. Verein. Limnol. 2, 1-38, 1958.

Adresse der Autoren: Dr. H.R. Bürgi, EAWAG, CH-8600 Dübendorf, Schweiz. Frl. B. Egli, Seenforschungslabor, $\mathrm{CH}-6047$ Kastanienbaum, Schweiz. 